\title{
HIV-1 low-level viraemia assessed with 3 commercial real-time PCR assays show high variability
}

Jean Ruelle ${ }^{1 *}$, Laurent Debaisieux ${ }^{2}$, Ellen Vancutsem³ ${ }^{3}$, Annelies De Bel ${ }^{3}$, Marie-Luce Delforge ${ }^{2}$, Denis Piérard ${ }^{3}$ and Patrick Goubau ${ }^{1}$

\begin{abstract}
Background: Current real-time PCR-based HIV-1 viral load (VL) assays allow the detection of residual viraemia in antiretroviral-treated patients. The clinical outcome of HIV-1 patients experiencing low-level replication $(<50 \mathrm{cop} / \mathrm{mL})$ in comparison with fully suppressed patients is currently debated. We analysed variability of $3 \mathrm{VL}$ assays $<50 \mathrm{cop} / \mathrm{mL}$, and evaluated the reproducibility of viral blips $<100 \mathrm{cop} / \mathrm{mL}$.
\end{abstract}

Methods: Three commercial VL assays were tested: Versant HIV-1 RNA 1.0 kPCR (Siemens), Abbott Realtime HIV-1, and Cobas Ampliprep/Cobas Taqman HIV-1 v2.0 (Roche). Ten replicates of a reference sample at 4 low target dilutions were tested to evaluate assay variability. Prospective collection of 181 clinical samples with detectable $V L<50 \mathrm{cop} / \mathrm{mL}$ was used to evaluate intra-and inter-assay variability by triplicate testing. Samples from 26 patients experiencing a viral blip were retested.

Results: All assays showed substantial variability at low VL level: the coefficient of variation at 100, 50, 25 and $12 \mathrm{cop} / \mathrm{mL}$ ranged respectively from 32 to $44 \%, 35$ to $68 \%, 41$ to $83 \%$ and 33 to $77 \%$. In the intra-assay evaluation of repeatability, 52.5 to $57.5 \%$ of detectable $V L<50 \mathrm{cop} / \mathrm{mL}$ tested in triplicate showed at least one fully undetected result. Variability was similar in the inter-assay arm. The VL blips could only be reproduced in 19\% of cases.

Conclusions: The most recent versions of widespread commercial VL assays showed substantial variability at low levels and residual viraemia could not be consistently reproduced. Patient outcome studies comparing residual VL to full suppression are therefore biased when using commercial assays.

Keywords: Residual viraemia, HIV-1 RNA, Viral load, Assay variability, Blip

\section{Background}

Plasma viral load (VL) measurements are part of the routine follow-up for patients infected with HIV [1,2]. Antiretroviral therapy (ART) aims to block viral replication, leading to plasma viraemia below the clinically validated threshold of 50 copies per millilitre (cop/mL) of plasma. Below this limit, the patient has the lowest morbidity and mortality probabilities [3]. However, this threshold of $50 \mathrm{cop} / \mathrm{mL}$ reflects the performance characteristics of VL assays available before the launch of real-time PCRbased assays, which allow the detection of low numbers of RNA copies present in the sample.

\footnotetext{
* Correspondence: jean.ruelle@uclouvain.be

${ }^{1}$ UCLouvain, AIDS Reference Laboratory, Avenue Hippocrate 54 - B1.54.05, 1200, Brussels, Belgium

Full list of author information is available at the end of the article
}

The limit of detection (LoD) does not usually equal the lower limit of quantification (LLoQ) at which the error meets the requirements for reproducibility and linearity [4]. Under the linear range, variability is unacceptably high and accurate quantification remains elusive [5,6]. Nonetheless, recent VL assays report qualitative results below LLoQ as detected or not detected, suggesting the presence or absence of residual viraemia.

The cause and the consequence of low $\mathrm{VL}$ are currently investigated [7]: HIV RNA can originate from the release of virus from the reservoirs or from residual replication [8]. Using modified protocols of commercial assays or inhouse assays to monitor VL, intensification of ART in patients with incompletely suppressed VL bore no result [9], and low-level viraemia was not associated with

\section{Biomed Central}


suboptimal CD4 gains during therapy [10]. Comparisons of drug efficacy on residual viraemia showed favourable profile for fixed-dose combinations including efavirenz [11], although others showed superior outcome when using nevirapine instead of efavirenz [12]. Some studies suggest that residual viraemia is predictive of future treatment failure [13], whilst some suggest no further effect [14]. We hypothesised that those results could not be translated to routine settings because they exceed the technical abilities of current commercial realtime PCR assays. We evaluated the variability of 3 commercial real-time HIV-1 VL assays on samples with detectable VL but $<50 \mathrm{cop} / \mathrm{mL}$, and analysed the repeatability of low-level viraemia in clinical routine settings in order to demonstrate the hypothesis.

\section{Results}

\section{Dilutions of a reference sample}

The descriptive statistics and the number of undetected samples are illustrated in Table 1. No signal was detected for any of the HIV-negative samples or negative kit controls throughout the study with any of the assays.

As noted during the preparation of the reference sample, the absolute quantification was the highest for the CAP/CTM v2.0, and the lowest for the kPCR that qualified $30 \%$ of the $100 \mathrm{cop} / \mathrm{mL}$ samples as detected below its LLoQ. At the target dilution of $12 \mathrm{cop} / \mathrm{mL}$, the Abbott Realtime HIV-1 assay showed the highest rate of

Table 1 Descriptive statistics related to 10 replicates of a reference sample at four target dilutions

\begin{tabular}{|c|c|c|c|c|}
\hline & & Siemens & Abbott & Roche \\
\hline \multirow[t]{4}{*}{$100 \mathrm{cop} / \mathrm{ml}$} & Mean $(\mathrm{cop} / \mathrm{mL})$ & 60.6 & 84.5 & 115.4 \\
\hline & $\overline{\mathrm{SD}}(\mathrm{cop} / \mathrm{mL})$ & 26.7 & 27.7 & 38.3 \\
\hline & CV (\%) & 44.0 & 32.8 & 33.2 \\
\hline & Undetected (\%) & 0 & 0 & 0 \\
\hline \multirow[t]{4}{*}{$50 \mathrm{cop} / \mathrm{ml}$} & Mean $(\mathrm{cop} / \mathrm{mL})$ & 35.3 & 33 & 65.8 \\
\hline & $\mathrm{SD}(\mathrm{cop} / \mathrm{mL})$ & 15.3 & 22.5 & 22.7 \\
\hline & $\overline{C V}(\%)$ & 43.2 & 68.1 & 34.5 \\
\hline & Undetected (\%) & 0 & 0 & 0 \\
\hline \multirow[t]{4}{*}{$25 \mathrm{cop} / \mathrm{ml}$} & Mean $(c o p / m L)$ & 18.1 & 25.5 & 26.56 \\
\hline & $\mathrm{SD}(\mathrm{cop} / \mathrm{mL})$ & 8.7 & 10.4 & 22.1 \\
\hline & $\overline{C V}(\%)$ & 48.1 & 40.7 & 83.1 \\
\hline & Undetected (\%) & 0 & 0 & 10 \\
\hline \multirow[t]{4}{*}{$12 \mathrm{cop} / \mathrm{ml}$} & Mean $(\mathrm{cop} / \mathrm{mL})$ & 15.6 & 20.0 & 25.8 \\
\hline & $\mathrm{SD}(\mathrm{cop} / \mathrm{mL})$ & 6.6 & 6.6 & 19.9 \\
\hline & CV (\%) & 42.2 & 32.8 & 77.1 \\
\hline & Undetected (\%) & 20 & 70 & 20 \\
\hline
\end{tabular}

Undetected samples were censored from the calculations. Siemens = Versant HIV-1 RNA 1.0 kPCR (Siemens), Abbott= Realtime HIV-1 (Abbott), Roche=Cobas Ampliprep/Cobas Taqman HIV-1 v2.0 (Roche), SD: standard deviation, CV: coefficient of variation. undetected samples with $70 \%$ of negative results while the others had $20 \%$ in comparison. Variability was the highest for CAP/CTM v2.0: the CV ranged from 33 to $83 \%$, while the CV of $\mathrm{kPCR}$ and Realtime assays varied respectively from 42 to $48 \%$, and from 32 to $68 \%$. Of note, CAP/CTM v2.0 has the highest variability at the 25 $\mathrm{cop} / \mathrm{mL}$ target and failed to detect one sample at a value theoretically above its LLoQ of 20, although below the LLoQ of the comparators. The distribution of values at each concentration is shown on Figure 1. Based on this set of results, the LoDs inferred by Pobit analysis were $14.76,18.89$ and $23.19 \mathrm{cop} / \mathrm{mL}$ respectively for the $\mathrm{kPCR}$, Abbott realtime and CAP/CTM v2.0, limit defined as the RNA concentration detected with $95 \%$ or greater probability.

\section{Clinical samples}

The variability was tested on 181 clinical samples obtained from patients experiencing residual viraemia $<50 \mathrm{cop} / \mathrm{mL}$. As samples had been selected prospectively and consecutively, with the only criterion defined as a detectable VL $<50 \mathrm{cop} / \mathrm{mL}$, we checked if the viral subtype was representative for our population. A genotype was available for $61.2 \%$ of the patients. The clade prevalence in our cohort was similar to the Belgian HIV-1 population, the most abundant ones being subtype $\mathrm{B}$, CRF02, A1 and C: respectively 47, 14, 14 and $10 \%$ of the patients.

Table 2 details the number of samples with discordant results between triplicates. All assays behaved similarly to over $50 \%$ of samples where at least one replicate was not detected. Between 2.5 and $5 \%$ of the samples had one replicate $>100 \mathrm{cop} / \mathrm{mL}$. Figure 2 details the distribution of the 61 samples tested by inter-assay comparison, as well as the distribution of 40 triplicates for each method in the intra-assay evaluation: variability is the cause of frequent results above the threshold of $50 \mathrm{cop} / \mathrm{mL}$, as well as undetected ones.

\section{Viral blips}

Medical records were examined and 26 cases corresponding to our blip definition (between 50 and 100 $\mathrm{cop} / \mathrm{mL}$ ) were re-analysed. Patients experiencing those blips represented between 1 and $1.5 \%$ of the population followed in the participating centres. The VL sequence of the blips, i.e. a VL $<50 \mathrm{cop} / \mathrm{mL}$ followed by a value between 50 and $100 \mathrm{cop} / \mathrm{mL}$ going back afterwards $<50$, could only be reproduced in 5 cases (19.2\%). In the remaining 21 cases, 16 were detected below $50 \mathrm{cop} / \mathrm{mL}$ and 5 were not detected. All samples preceding and following the putative blip gave a $<50 \mathrm{cop} / \mathrm{mL}$ result again, except for one case where the value before the blip and the blip itself were respectively 62 and $64 \mathrm{cop} / \mathrm{mL}$. 


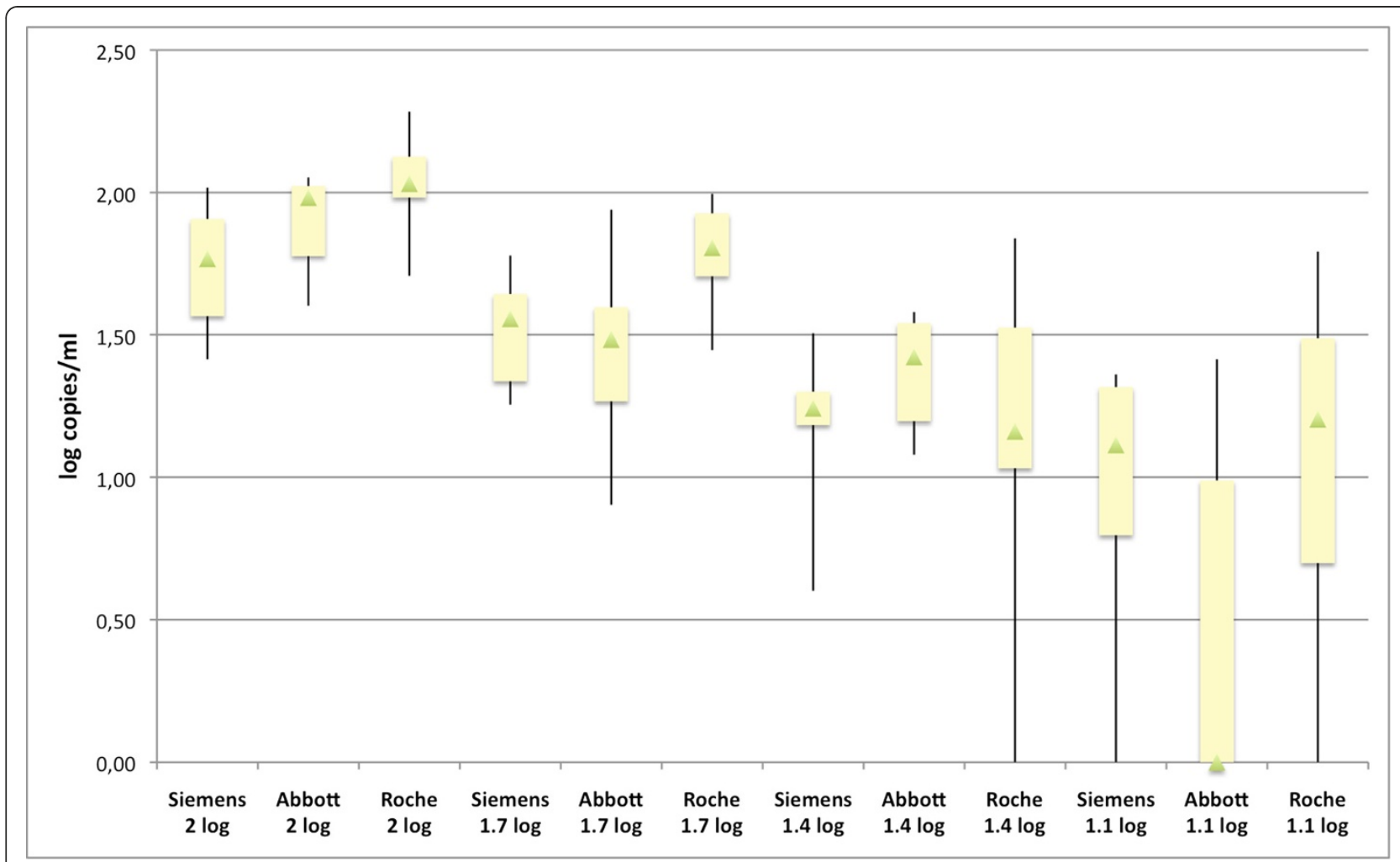

Figure 1 Low-level VL variability evaluated with a reference sample. Box Plot showing the distribution of 10 replicates of a reference sample at four dilutions $(100,50,25$ and $12 \mathrm{cop} / \mathrm{ml}$ or 2, 1.7, 1.4 and $1.1 \mathrm{log} \mathrm{cop} / \mathrm{ml})$ tested on each platform. The bottom and the top of the box represent the lower and the upper quartiles respectively. The triangle in the box is the median, and the ends of the whiskers correspond to the minimum and the maximum values. Siemens =Versant HIV-1 RNA $1.0 \mathrm{kPCR}$ (Siemens), Abbott = Realtime HIV-1 (Abbott), Roche =Cobas Ampliprep/Cobas Taqman HIV-1 v2.0 (Roche).

\section{Discussion}

Variability of three commercial HIV-1 VL assays was investigated at low values below the clinical cut-off of $50 \mathrm{cop} / \mathrm{mL}$.

As all the assays tested used automated platforms for viral RNA extraction and PCR reactive handling, no discrepancies due to human interference are to be considered. Moreover, the samples underwent no additional thaw and freeze cycle. Depending on the assay, variability at $50 \mathrm{cop} / \mathrm{mL}$ rose from 34 to $68 \%(\mathrm{CV})$ when a reference sample was diluted in human HIV-negative plasma: results round that cut-off are therefore uncertain, making a precise estimation of values just above $50 \mathrm{cop} / \mathrm{mL}$ difficult. Even at the $100 \mathrm{cop} / \mathrm{mL}$ target, 30\% of samples were quantified $<$ LLoQ with the $\mathrm{kPCR}$ assay. When focussing on lower values, variability was the highest with the CAP/CTM v2.0 assay. The latter missed 1 out of 10 replicates above its LLoQ. The Abbott Realtime

Table 2 Intra- and inter-assay evaluation of repeatability with clinical samples from patients experiencing residual viraemia

\begin{tabular}{|c|c|c|c|c|c|}
\hline & $\begin{array}{l}\% \text { of samples } \\
\text { where at } \\
\text { least one } \\
\text { replicate is } \\
\text { not detected }\end{array}$ & $\begin{array}{l}\% \text { of } \\
\text { samples } \\
\text { where only } \\
\text { one triplicate } \\
\text { is detected }\end{array}$ & $\begin{array}{l}\% \text { of samples } \\
\text { where at least } \\
\text { one replicate } \\
\text { was higher } \\
\text { than } 50 \mathrm{cop} / \mathrm{mL}\end{array}$ & $\begin{array}{l}\% \text { of samples } \\
\text { where at least } \\
\text { one replicate } \\
\text { was higher } \\
\text { than } 100 \mathrm{cop} / \mathrm{mL}\end{array}$ & $\begin{array}{l}\% \text { of samples } \\
\text { where at least } \\
\text { one replicate } \\
\text { was higher } \\
\text { than } 200 \mathrm{cop} / \mathrm{mL}\end{array}$ \\
\hline Intra-assay Siemens $(N=40)$ & 52.5 & 32.5 & 2.5 & 2.5 & 0.0 \\
\hline Intra-assay Abbott(N=40) & 57.5 & 20.0 & 15.0 & 5.0 & 0.0 \\
\hline Intra-assay Roche $(\mathrm{N}=40)$ & 55.0 & 20.0 & 12.5 & 2.5 & 0.0 \\
\hline Inter-assay $(\mathrm{N}=61)$ & 52.5 & 13.1 & 18.0 & 4.9 & 3.3 \\
\hline
\end{tabular}

Each sample was tested in triplicate, the first replicate being detected but quantified below 50 cop/mL. Siemens =Versant HIV-1 RNA $1.0 \mathrm{kPCR}$ (Siemens), Abbott = Realtime HIV-1 (Abbott), Roche = Cobas Ampliprep/Cobas Taqman HIV-1 v2.0 (Roche). 

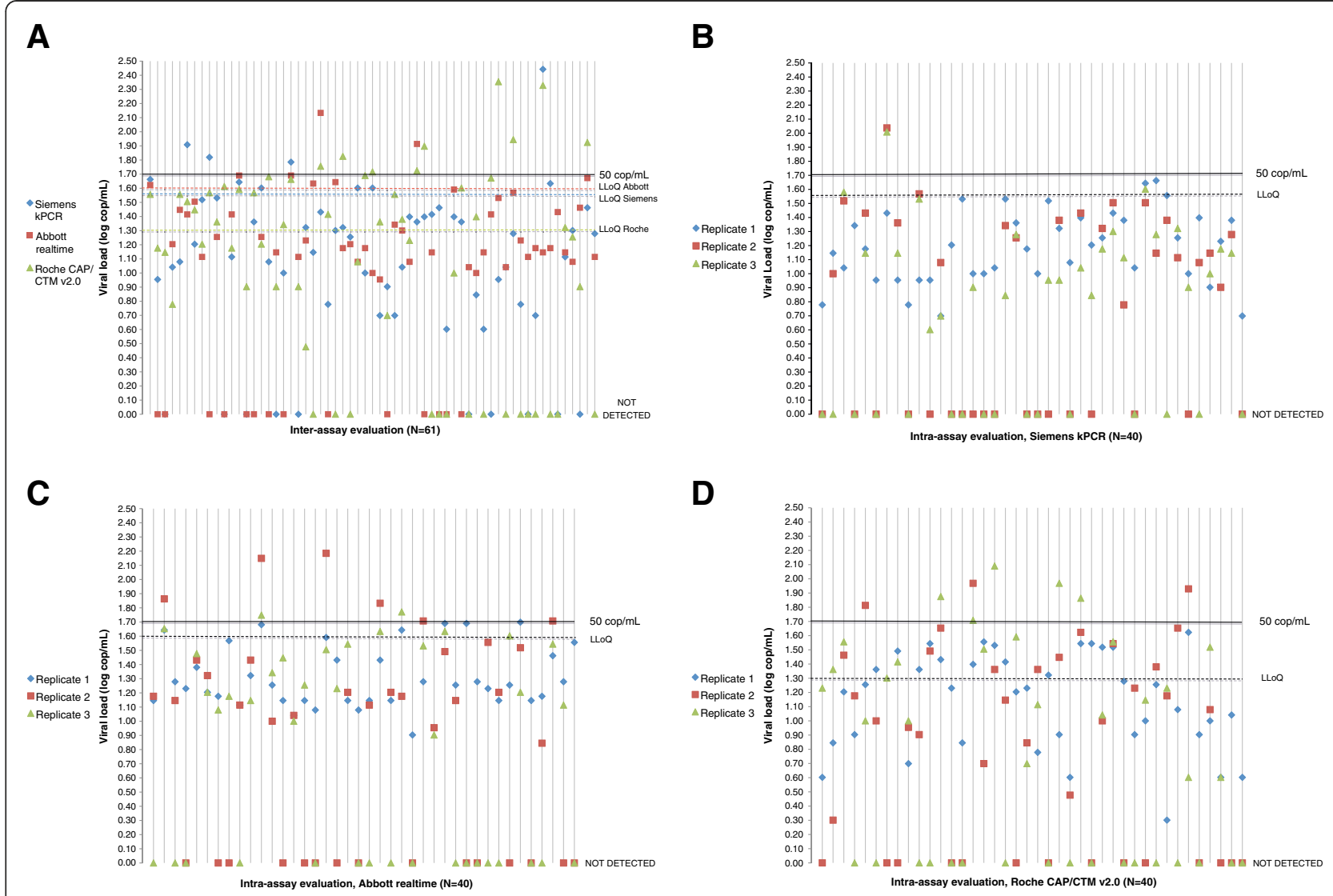

Figure 2 Repeatability of VL below $\mathbf{5 0} \mathrm{cop} / \mathrm{ml}$ in clinical settings. A. Inter-assay variability. The plasma VL of sixty-one samples were tested with the assays from Siemens=Versant HIV-1 RNA 1.0 kPCR (Siemens), Abbott=Realtime HIV-1 (Abbott), Roche =Cobas Ampliprep/Cobas Taqman HIV-1 v2.0 (Roche). B. Intra-assay repeatability on 40 triplicates tested with Versant HIV-1 RNA 1.0 kPCR (Siemens). C. Intra-assay repeatability on 40 triplicates tested with Realtime HIV-1 (Abbott). D. Intra-assay repeatability on 40 triplicates tested with Cobas Ampliprep/Cobas Taqman HIV-1 v2.0 (Roche).

HIV-1 assay seemed less sensitive than the others at the $12 \mathrm{cop} / \mathrm{mL}$ level, although the LoDs inferred from our results are similar with the 3 assays, ranging from 15 to $23 \mathrm{cop} / \mathrm{mL}$.

Repeatability of RNA detection $<50 \mathrm{cop} / \mathrm{mL}$ was evaluated on 181 triplicates, either intra- or inter-assay: more than $50 \%$ of results cannot be reproduced on the 3 aliquots.

Those observations are in line with the PCR limits, as well as with the limits given by the assay manufacturers: every PCR will lead to stochastic results when targets introduced in the reaction mixture lie between the limit of blank and the limit of detection [4], and companies define their own assay lowest LLoQ level below which the results cannot be treated in a quantitative manner. Extreme values cannot be reproduced quantitatively and are therefore considered as "lower than LLoQ" in clinical practice.

The results below the LLoQ are reported as detected or not detected by the assays software, and such information should not be reported to the clinician because of uncertainty. This distinction between the presence and absence of PCR signal, together with the launch of assays with LoQ below $50 \mathrm{cop} / \mathrm{mL}$, led to the notion that some patients previously considered as therapy-controlled undergo low-level viraemia, and that others are fully suppressed, i.e. no viral genome can be detected in the plasma. Studies investigating patient outcome when residual viraemia is detected were published, as well as intensification trials exploring the effect of an additional antiretroviral drug on full viral suppression. Some authors used methodology enabling to concentrate the HIV-1 RNA by extracting from $4 \mathrm{~mL}$ up to $30 \mathrm{~mL}$ of plasma following ultracentrifugation [15-17], or repeat the test 3 times [18], lowering the detection limits of PCR and reducing the variability in the low copy number range. Those modified assays may be useful to answer scientific questions about low-level viraemia, but cannot be translated into clinical practice because the variability of most recent versions of widespread commercial VL assays is too high.

Our study was not designed to study the assays' specificities. Previous studies showed that HIV-negative 
samples do not lead to false positive signal, as confirmed here with 10 negative samples. Low values can indeed be considered as true viraemia [19] but negative ones cannot be considered as proof of full viral suppression. RNA viral genomes can originate from long-living infected cells producing viral particles in the absence of replication, or reflect a residual replication. Strategies aiming at viral eradication need to target the source of circulating viruses [20]. Whether this residual viraemia is the beginning of virological failure is subject to debate $[7,13,14]$. We would suggest that current commercial real-time PCR assays do not have sufficient precision to answer this burning question. Variability in the low copy number range has also implications both for the classification of elite controllers as well as for diagnosis strategies using molecular assays.

In the same way, outcome comparisons of different ART regimens regarding full suppression must be powered enough to take into account assay variability. Although the samples used in this study were taken from patients on suppressive ART, the population was not big enough to detail the drug classes used in the subgroup of reproducible low VL versus the others.

Viral blips between 50 and $100 \mathrm{cop} / \mathrm{mL}$ were not reproduced in $80 \%$ of the 26 cases. As analytical assay variability is high around $50 \mathrm{cop} / \mathrm{mL}$ (Figure 1), it is therefore useful in clinical practice to confirm the result on a new sample to assess if viral replication is really increasing: the samples following the blips tested here were all repeatedly $<50 \mathrm{cop} / \mathrm{mL}$. Our results are in line with the 2011 revision of the DHHS guidelines which define the virological failure threshold as $200 \mathrm{cop} / \mathrm{mL}$ [1]. Consequently, a single value between 50 and $200 \mathrm{cop} / \mathrm{mL}$ should not be a reason enough for dropping a case out of clinical studies.

\section{Conclusions}

In conclusion, state of the art VL assays tested here showed poor reproducibility of HIV-1 low-level viraemia results. When used in clinical routine settings with single measurements, they introduce biases in patient outcome studies comparing very low VL to full viral suppression.

\section{Methods}

\section{Participating laboratories and assays}

Three AIDS reference laboratories in Brussels accredited by national legal bodies to either the EN-ISO 15189 or the EN-ISO 17025 standard performed the analyses, one assay running per site. The assays tested were Versant HIV-1 RNA $1.0 \mathrm{kPCR}$ (Siemens, performed at UCLouvain, using $0.5 \mathrm{~mL}$ of plasma), Realtime HIV-1 (Abbott, performed at Erasme using the $0.6 \mathrm{~mL}$ plasma volume protocol), and Cobas Ampliprep/Cobas Taqman HIV-1 v2.0 (CAP/CTM, Roche Diagnostics, perfomed at UZ Brussel, using $0.85 \mathrm{~mL}$ of plasma). The manufacturers respectively report the LLoQs as 37,40 and $20 \mathrm{cop} / \mathrm{mL}$. The volumes of plasma mentioned are those actually extracted by the platforms, excluding the dead volume linked to the pipetting steps. Only a part of the RNA is introduced in the RT-PCR reaction: respectively $79 \%, 56 \%$ and $67 \%$ of the total elution volume, corresponding to $0.39,0.33$ and $0.57 \mathrm{~mL}$ of plasma.

The manufacturers' recommendations were strictly followed and the same reagent lot was used throughout the study, except for the re-evaluation of blips.

We did obtain quantitative results beyond the assays' LLoQs using the $\mathrm{Cq}$ and the calibration curve and the manufacturing companies offered some help when the assay software did not produce a value in IVD conditions.

\section{Dilutions of a reference sample}

A reference HIV-1 sample, i.e. a culture supernatant dilution of a subtype $B$ isolate, is routinely used in all of the Belgian centres as an internal control in each VL run. We assigned a "true" value to that reference sample based on the results obtained in the last $25 \mathrm{VL}$ runs per assay: the mean VL \pm SD was $3.13 \pm 0.12$ (Siemens), $3.18 \pm 0.07$ (Abbott) and $3.37 \pm 0.08 \log _{10} \mathrm{cop} / \mathrm{mL}$ (Roche). The average value of $3.23 \log _{10} \mathrm{cop} / \mathrm{mL}$ was used to prepare aliquots of 100, 50, 25 and $12 \mathrm{cop} / \mathrm{mL}(2,1.7,1.4$ and $1.1 \log \mathrm{cop} / \mathrm{mL}$ ) in commercial HIV-negative human plasma (A\&E Scientific, Belgium). Participating laboratories blind tested 10 aliquots of each dilution as well as 10 aliquots of the negative plasma used to prepare the samples. The LoD was inferred by probit analysis using the IBM SPSS Statistics 20 software.

\section{Variability in patients with residual viraemia}

The variability in clinical settings with patients experiencing residual viraemia below $50 \mathrm{cop} / \mathrm{mL}$ was assessed with 181 samples. Each laboratory selected prospectively and consecutively 62 plasma samples: when a fresh whole EDTA blood sample was received for HIV-1 VL testing, 3 aliquots were frozen and stored below $-70^{\circ} \mathrm{C}$. If the first aliquot used for routine analysis gave a detectable result $<50 \mathrm{cop} / \mathrm{mL}$, the sample was included. Apart from this condition and the availability of sufficient sample volume, no selection for the inclusion of samples was made. Only one sample per patient was included. The maximal duration of storage was limited to 6 weeks and no additional freeze/thaw cycles were allowed. Forty samples were tested in triplicate on the same platform during different runs to assess the intra-assay variability, and 22 others were shared with the 2 other laboratories for an inter-assay evaluation. If one replicate gave an invalid result, the sample was excluded from the analysis: 61 samples were finally analysed in the inter-assay evaluation whilst 5 were excluded because of failure of 1 replicate. The 
TRUGENE HIV-1 genotyping kit (Siemens, Tarrytown, NY) determined the HIV-1 subtype based on the reverse transcriptase sequence.

\section{Viral blips}

Laboratories searched their records to identify patients who experienced a blip, i.e. a VL between 50 and 100 $\mathrm{cop} / \mathrm{mL}$ during the follow-up and their previous and next sample was $<50 \mathrm{cop} / \mathrm{mL}$, during the last 15 months. The median time between the first and the second analysis was 7 months (ranging from 3 to 15), plasma samples being stored at $-80^{\circ} \mathrm{C}$. Twenty-six blips, together with the previous and next sample from that patient, were retested with the same assay and without additional thaw and freeze cycle.

\section{Abbreviations \\ VL: Viral load; ART: antiretroviral therapy; cop/mL: copies/mL; LoD: limit of detection; LLoQ: lower limit of quantification; Cq: cycle of quantification; CAP/CTM: Cobas Ampliprep/Cobas Taqman HIV-1 v2.0; CV: coefficient of variation; IVD: in vitro diagnostic}

\section{Competing interests}

JR was invited twice as a speaker for Siemens Healthcare Diagnostics. The other authors have declared no competing interests.

\section{Authors' contributions}

$J R$ initiated the study, centralised the data and drafted the manuscript. LD, EV and ADB participated in the study design and helped to draft the manuscript. MLD, DP and PG revised the design of the draft and provided critical input. All authors read and approved the final manuscript.

\section{Acknowledgements}

The authors would like to acknowledge laboratory technicians Anita Wyns, Veerle Bastiaensen and Linda Godau at the UZ Brussel, Isabelle Lefèvre, Najet Lamarti and Philippe de Sany at the UCLouvain, and Maria-Helena Jurion, Karin Miller and Nadine Gijbels at the Erasme Hospital.

The 3 companies involved in the present evaluation provided free kits, while Abbott and Roche provided support to infer quantitative results under their respective LLOQs.

\section{Author details}

${ }^{1}$ UCLouvain, AIDS Reference Laboratory, Avenue Hippocrate 54 - B1.54.05, 1200, Brussels, Belgium. ${ }^{2}$ Hôpital Universitaire Erasme, AIDS Reference Laboratory, Route de Lennik 808, 1070, Brussels, Belgium. ${ }^{3}$ Vrije Universiteit Brussel, AIDS Reference Laboratory, site Universitair Ziekenhuis Brussel, Laarbeeklaan 101, 1090, Brussels, Belgium.

Received: 5 January 2012 Accepted: 24 April 2012

Published: 24 April 2012

\section{References}

1. Panel on Antiretroviral Guidelines for Adults and Adolescents: Guidelines for the use of antiretroviral agents in HIV-1-infected adults and adolescents. Department of Health and Human Services 1-239. Available at http://aidsinfo.nih.gov/ContentFiles/AdultandAdolescentGL.pdf.

2. European AIDS Clinical Society Guidelines Version 6.0 [www.eacs.eu]

3. Cohen C: Low-level viremia in HIV-1 infection: consequences and implications for switching to a new regimen. HIV Clin Trials 2009, 10:116-124.

4. Protocols for Determination of Limits of Detection and Limits of Quantification. In Approved guideline NCCLS document EP17-A. NCCLS, Wayne, Pennsylvania; 2004.

5. Paba P, Fabeni L, Ciccozzi M, Perno CF, Ciotti M: Performance evaluation of the COBAS/TaqMan HIV-1 v2.0 in HIV-1 positive patients with low viral load: a comparative study. J Virol Methods 2011, 173:399-402.

6. Yan CS, Hanafi I, Kelleher AD, Carr AD, Amin J, McNally LP, Cunningham PH: Lack of correlation between three commercial platforms for the evaluation of human immunodeficiency virus type 1 (HIV-1) viral load at the clinically critical lower limit of quantification. J Clin Virol 2010, 49:249-253.

7. Pozniak A, Gupta RK, Pillay D, Arribas J, Hill A: Causes and consequences of incomplete HIV RNA suppression in clinical trials. HIV Clin Trials 2009, 10:289-298

8. Mavigner M, Delobel P, Cazabat M, Dubois M, L'Faqihi-Olive FE, Raymond S, Pasquier C, Marchou B, Massip P, Izopet J: HIV-1 residual viremia correlates with persistent T-cell activation in poor immunological responders to combination antiretroviral therapy. PLoS One 2009, 4:e7658.

9. Gandhi RT, Zheng L, Bosch RJ, Chan ES, Margolis DM, Read S, Kallungal B, Palmer S, Medvik K, Lederman MM, et al: The effect of raltegravir intensification on low-level residual viremia in HIV-infected patients on antiretroviral therapy: a randomized controlled trial. PLOS Med 2010, 7:8.

10. Hatano H, Hayes TL, Dahl V, Sinclair E, Lee TH, Hoh R, Lampiris H, Hunt PW, Palmer S, McCune JM, et al: A randomized, controlled trial of raltegravir intensification in antiretroviral-treated, HIV-infected patients with a suboptimal CD4+ T cell response. J Infect Dis 2011, 203:960-968.

11. Rosso R, Di Biagio A, Maggiolo F, Nulvesu L, Callegaro AP, Taramasso L, Bruzzone B, Viscoli C: Patient-reported outcomes and low-level residual HIV-RNA in adolescents perinatally infected with HIV-1 after switching to one-pill fixed-dose regimen. AIDS Care 2011, 24:54-58.

12. Haim-Boukobza S, Morand-Joubert L, Flandre P, Valin N, Fourati S, Sayon S, Lavignon M, Simon A, Girard PM, Katlama C, et al: Higher efficacy of nevirapine than efavirenz to achieve HIV-1 plasma viral load below 1 copy/ml. AIDS 2011, 25:341-344.

13. Widdrington J, Payne B, Medhi M, Valappil M, Schmid ML: The significance of very low-level viraemia detected by sensitive viral load assays in HIV infected patients on HAART. J Infect 2011, 62:87-92.

14. Gianotti N, Galli L, Racca S, Salpietro S, Cossarini F, Spagnuolo V, Barda B, Canducci F, Clementi M, Lazzarin A, Castagna A: Residual viraemia does not influence 1 year virological rebound in HIV-infected patients with HIV RNA persistently below 50 copies/mL. J Antimicrob Chemother 2011, 67:213-217.

15. Yukl SA, Li P, Fujimoto K, Lampiris H, Lu CM, Hare CB, Deeks SG, Liegler T, Pandori M, Havlir DV, Wong JK: Modification of the Abbott RealTime assay for detection of HIV-1 plasma RNA viral loads less than one copy per milliliter. J Virol Methods 2011, 175:261-265.

16. Amendola A, Bloisi M, Marsella P, Sabatini R, Bibbo A, Angeletti C, Capobianchi MR: Standardization and performance evaluation of "modified" and "ultrasensitive" versions of the Abbott RealTime HIV-1 assay, adapted to quantify minimal residual viremia. J Clin Virol 2011, 52:17-22.

17. Anderson JA, Archin NM, Ince W, Parker D, Wiegand A, Coffin JM, Kuruc J, Eron J, Swanstrom R, Margolis DM: Clonal sequences recovered from plasma from patients with residual HIV-1 viremia and on intensified antiretroviral therapy are identical to replicating viral RNAs recovered from circulating resting CD4+ T cells. J Virol 2011, 85:5220-5223.

18. Mens H, Kearney M, Wiegand A, Spindler J, Maldarelli F, Mellors JW, Coffin JM: Amplifying and Quantifying HIV-1 RNA in HIV Infected Individuals with Viral Loads Below the Limit of Detection by Standard Clinical Assays. J Vis Exp 2011, 55:e2960.

19. Verhofstede C, Van Wanzeele F, Reynaerts J, Mangelschots M, Plum J, Fransen K: Viral load assay sensitivity and low level viremia in HAART treated HIV patients. J Clin Virol 2010, 47:335-339.

20. Chun TW, Murray D, Justement JS, Hallahan CW, Moir S, Kovacs C, Fauci AS: Relationship between residual plasma viremia and the size of HIV proviral DNA reservoirs in infected individuals receiving effective antiretroviral therapy. J Infect Dis 2011, 204:135-138.

doi:10.1186/1471-2334-12-100

Cite this article as: Ruelle et al: HIV-1 low-level viraemia assessed with 3 commercial real-time PCR assays show high variability. BMC Infectious Diseases 2012, 12:100 